\title{
EFFECT OF EXPERIMENTAL HYPERHOMOCYSTEINEMIA ON PLASMA LIPID PROFILE, INSULIN SENSITIVITY AND PARAOXONASE 1 IN THE RAT
}

\author{
Jerzy Bełtowski ${ }^{1}$, Grażyna Wójcicka ${ }^{1}$, and Andrzej Wojtak ${ }^{2}$ \\ 'Department of Pathophysiology, Medical University, and 'Department of Vascular Surgery, Medical University, \\ Lublin, Poland
}

\begin{abstract}
Hyperhomocysteinemia ( $\mathrm{hHcy}$ ) is a well-known risk factor of cardiovascular diseases, however, the mechanism of its detrimental effect is incompletely understood. Some studies suggest that, paradoxically, hHcy may promote traditional risk factors such as hyperlipidemia. We examined the effect of experimental hHcy on plasma lipid profile, glucose and insulin concentrations as well as on high-density lipoprotein (HDL)-associated antiatherosclerotic enzyme, paraoxonase 1 (PON1). Hyperhomocysteinemia was induced by feeding male Wistar rats with diet enriched with methionine or diet enriched in methionine and deficient in folate, vitamin $\mathrm{B}_{6}$ and $\mathrm{B}_{12}$ for 8 weeks. These diets resulted in the 3.3- and 9.6-fold elevation of plasma Hcy, respectively. Plasma total and HDL-cholesterol, triglycerides and apolipoprotein A-I were similar in all groups. Highmethionine diets had no effect on fasting plasma glucose but significantly increased fasting plasma insulin concentration indicating impaired ability of insulin to suppress hepatic glucose output. PON1 activity was unchanged in high methionine vitamin Bsufficient diet-fed rats, but was decreased by $30-40 \%$ toward various substrates in high methionine vitamin B-deficient diet-fed animals. The results indicate that hHcy has no effect on lipid metabolism, however, induces insulin resistance and, above certain Hcy level, PON1 deficiency. Impaired insulin signaling and reduced PON1 activity may contribute to detrimental effects of hHcy.
\end{abstract}

Adipobiology 2012; 4: 77-84

Key words: homocysteine, plasma lipoproteins, hyperlipidemia, paraoxonase, adipose tissue, insulin resistance, lipolysis, atherosclerosis

Received 12 September 2012, accepted 18 September 2012.

Correspondence and reprint request to Dr Jerzy Bettowski, Department of Pathophysiology, Medical University, ul. Jaczewskiego 8, PL-20-090 Lublin, Poland. Tel.: +48 81 7187365; Fax: +48 81 7187364; E-mail: jerzy.beltowski@umlub.pl; jerzybel@hotmail.com

\section{Introduction}

Homocysteine (Hcy) is a nonprotein aminoacid which is synthesized from dietary methionine through S-adenosylmethionine and S-adenosylhomocysteine as intermediates, and is metabolized by either remethylation to methionine by methionine synthase or by transsulfuration to cysteine by cystathionine $\beta$-synthase (CBS) and cystathionine $\gamma$-lyase (CSE) (1). Hcy metabolism is dependent on the availability of folate (its derivative, 5-methyltetrahydrofolate, donates methyl groups for Hcy remethylation), vitamin $B_{12}$ (a cofactor of 5-methyltetrahydrofolate synthesis) and vitamin $\mathrm{B}_{6}$ (a cofactor of both CBS and CSE) (2). Normal plasma Hcy concentration is about 5-15 $\mu \mathrm{M}$. Many clinical studies have demonstrated that hyperhomocysteinemia (hHcy) is an independent risk factor of atherosclerosis and thrombosis. In addition, hyperhomocysteinemia is involved in the pathogenesis of many other disorders including glomerulopathy, Alzheimer disease, neural tube defects and osteoporosis. Hyperhomocysteinemia 
may be responsible for high risk of cardiovascular diseases in many patients without classical atherosclerosis risk factors (e.g. those with normal blood pressure, normolipidemia and normoglycemia) as well as for the residual risk in patients treated with anti-classical risk factor therapies such as cholesterol-lowering statins, antidabetic and antihypertensive medications. The most important causes of hyperhomocysteinemia are genetic defects of Hcy-metabolizing enzymes such as CBS or methylenetetrahydrofolate reductase (MTHFR), high methionine intake (e.g. diet rich in meat), deficiency of folate, vitamin $\mathrm{B}_{6}$ and $\mathrm{B}_{12}$, metabolic syndrome and renal dysfunction. In addition, hyperhomocysteinemia may be induced or aggravated by certain drugs including those used to prevent cardiovascular diseases such as fibrates (2).

Although proatherogenic effect of hHcy is well established, the mechanism of detrimental impact of homocysteine is unclear. Several effects of Hcy have been described such as unbeneficial shift of coagulation/fibrinolysis balance, hypertrophy of vascular smooth muscle cells, induction of vascular inflammation, oxidative stress and depletion of vascular nitric oxide (3). However, most of these effects were observed using high, supraphysiological or even "suprapathological" concentrations of Hcy such as 1-10 mM, whereas plasma Hcy level even in severe hHcy rarely exceeds $200 \mu \mathrm{M}$. Therefore, the molecular target for Hcy is still searched for.

Several studies have demonstrated that hHcy may have a detrimental effect on plasma lipid profile. However, these studies are either observational clinical correlation studies which cannot imply the cause-effect relationship, or experiments in specific animal models such as CBS knockout mice or hHcy induced by very high methionine intake, which may have effects independent of Hcy elevation. In addition, insulin resistance and metabolic syndrome are well established risk factors of atherosclerosis. The association between hHcy and insulin resistance/ metabolic syndrome has been observed, but it is unclear if insulin resistance results in hyperhomocysteinemia or, vice versa, homocysteine impairs insulin signaling. In the present study we examined the effect of hHcy induced in the rat by moderate increase in methionine intake on plasma lipid profile as well as on glucose and insulin concentrations. In addition, we measured plasma paraoxonase 1 (PON1) activity. PON1 is a high-density lipoprotein (HDL)-associated atheroprotective enzyme which decomposes lipid peroxidation products as well as homocysteine thiolactone - the toxic endogenous Hcy derivative (4). Atherosclerosis is aggravated and reduced in PON1 knockout and PON1-overexpressing mice, respectively and low PON1 is a risk factor of cardiovascular events in humans. Changes in plasma lipid profile, especially low HDL, is often associated with
PON1 deficiency (4). Therefore, it is of interest if hHcy affect PON1 status.

\section{Materials and methods}

The study was performed on adult male Wistar rats weighing 264 $\pm 6 \mathrm{~g}$ before the experiment. We used the experimental model of hHcy described by De Vriese et al (5). After 2-week acclimation, animals were randomized to three experimental groups $(n=8$ each): (1) control, fed standard rodent chaw (2) high-methionine diet (Harlan Teklad TD98002), (3) high-methionine diet deficient in folate, vitamin $\mathrm{B}_{6}$ and $\mathrm{B}_{12}$ (Harlan Teklad TD97345). Except methionine and vitamins, the content of other nutrients was identical in all diets. The composition of diets is presented in Table 1. The respective diets were administered for 8 weeks.

Table 1. Composition of diets in different experimental groups

\begin{tabular}{|l|l|l|l|}
\hline Group & Control & $\begin{array}{l}\text { High } \\
\text { methionine }\end{array}$ & $\begin{array}{l}\text { High } \\
\text { methionine } \\
\text { vitamin B } \\
\text { deficient }\end{array}$ \\
\hline Methionine $(\mathrm{g} / \mathrm{kg})$ & 3.8 & 7.7 & 7.7 \\
\hline Folic acid $(\mathrm{g} / \mathrm{kg})$ & 0.002 & 0.002 & - \\
\hline Vitamin $\mathrm{B}_{6}(\mathrm{~g} / \mathrm{kg})$ & 0.07 & 0.07 & - \\
\hline Vitamin $\mathrm{B}_{12}(\mathrm{~g} / \mathrm{kg})$ & 0.00003 & 0.00003 & - \\
\hline Choline $(\mathrm{g} / \mathrm{kg})$ & 2.5 & 2.5 & 2.5 \\
\hline
\end{tabular}

Blood for biochemical studies was withdrawn after the 6-hour fasting. Rats were anesthetized with thiopental $(50 \mathrm{mg}$ / kg ip.) and blood was withdrawn from the abdominal aorta into heparinized tubes (for PON1 assay) and EDTA-containing tubes (for the remaining assays). Plasma was separated by centrifugation at $3000 \mathrm{rpm}$ for $10 \mathrm{~min}$ at $4^{\circ} \mathrm{C}$, frozen and stored at $-80^{\circ} \mathrm{C}$ until analysis.

Total plasma Hcy was measured by enzyme immunoassay using kit purchased from Axis Shield Diagnostics (Dundee, UK). Since most of the plasma Hcy occurs in the form of disulfides, samples were first reduced with dithiothreitol (DTT) to convert Hcy to a free thiol form. Hcy is then converted to S-adenosylhomocysteine (SAH) by adding the excess of adenosine and SAH hydrolase, and the latter is assayed using specific anti-SAH antibodies (6). PON1 activity toward synthetic (par- 
aoxon and phenyl acetate) as well as natural substrate (homocysteine thiolactone) was measured as described previously (6).

Total plasma cholesterol, HDL-cholesterol, triglycerides and glucose were measured spectrophotometrically using commercially available kits (AlphaDiagnostics, Warsaw, Poland). Alanine and aspartate aminotransferase (ALT and AST) activities were measured using Sigma-Aldrich kits and are expressed in Sigma-Frankel units/ml. Insulin and apolipoprotein A-I concentrations were measured by enzyme-linked immunoassay using kits specific for rat proteins (Mercodia, Uppsala, Sweden and Uscnlife, East Lack, China, respectively); insulin concentration calculated from standard curve in $\mu \mathrm{g} / \mathrm{l}$ was expressed in $\mu \mathrm{U} / \mathrm{ml}$ assuming WHO definition: $1 \mathrm{mg}=26 \mathrm{U}$. Plasma non-esterified fatty acids (NEFA) and glycerol were measured by spectrofluorometric and spectrophotometric methods, respectively, using Cayman Chemical kits. For details of these methods, see (7). Homeostatic Model Assessment-Insulin Resistance Index (HOMA-IR) was calculated as: $\mathrm{I} \times \mathrm{G} / 22.5$, where $\mathrm{I}$ is fasting plasma insulin in $\mu \mathrm{U} / \mathrm{ml}$ and $\mathrm{G}$ is fasting plasma glucose in $\mathrm{mM}$. Quantitative Insulin Sensitivity Check Index (QUICKI) was calculated as $1 /[\log (G)+\log (\mathrm{I})]$, where $G$ is fasting plasma glucose in $\mathrm{mg} / \mathrm{dl}$ and $\mathrm{I}$ is fasting plasma insulin in $\mu \mathrm{U} / \mathrm{ml}$. HOMA-IR and QUICKI, derived from fasting plasma insulin and glucose, reflect mainly the ability of insulin to suppress hepatic glucose output (8).

Results are expressed as mean \pm SEM from 8 animals in each group. Statistical analysis was performed by one-way ANOVA followed by Tukey post-hoc test. A p value $<0.05$ was considered significant.

\section{Results}

Administration of methionine-rich or methionine-rich vitamin $B$ deficient diets had no effect on food intake (not shown) and weight gain of the animals (Table 2). Plasma Hcy concentration was higher $(\mathrm{p}<0.001)$ in high methionine-rich diet fed rats $(26.4 \pm 2.5 \mu \mathrm{M})$ than in control animals $(7.9 \pm 0.5 \mu \mathrm{M})$. In group receiving high-methionine vitamin B-deficient diet plasma Hcy was $76.1 \pm 9.3 \mu \mathrm{M}$, i.e. significantly higher than in control $(\mathrm{p}<0.001)$ and methionine-rich diet fed rats $(\mathrm{p}<0.05)$.

Plasma concentrations of triglycerides, total cholesterol, HDL-cholesterol and apolipoprotein A-I were not different between groups. In addition, hHcy induced by either diet had no effect on alanine and aspartate aminotransferase activities (Table 2).

Plasma glucose concentration was similar in all groups. In contrast, both methionine-rich diets increased fasting plasma insulin concentration. However, insulin concentration did not differ between methionine-fed groups with different vitamin B content (Table 3). Consequently, HOMA-index, a marker of insulin resistance, was higher in methionine-enriched and methionine-enriched vitamin B-deficient diet fed rats by $73.6 \%$ and 91.9\%, respectively; the difference between both hHcy groups being non-significant. On the contrary, quantitative insulin sensitivity check index was lower in rats fed methionine-enriched and methionine-enriched vitamin B-deficient diets by $6.6 \%$ and $7.9 \%$, respectively (Table 3 ). Plasma concentrations of glycerol and non-esterified fatty acids were not different between groups (Table 3).

Plasma PON1 activity in rats fed methionine-enriched diet

Table 2. Effect of hyperhomocysteinemia on body weight, plasma lipids and liver enzymes

\begin{tabular}{|l|l|l|l|}
\hline Group & Control & High methionine & $\begin{array}{l}\text { High methionine } \\
\text { vitamin B deficient }\end{array}$ \\
\hline Body weight $(\mathrm{g})$ & $345 \pm 11$ & $352 \pm 9$ & $368 \pm 11$ \\
\hline Triglycerides (mM) & $0.63 \pm 0.07$ & $0.61 \pm 0.06$ & $0.69 \pm 0.08$ \\
\hline Total cholesterol (mM) & $1.64 \pm 0.19$ & $1.71 \pm 0.20$ & $1.76 \pm 0.17$ \\
\hline HDL-cholesterol (mM) & $0.77 \pm 0.07$ & $0.83 \pm 0.08$ & $0.79 \pm 0.06$ \\
\hline Apolipoprotein A-I (mg/dl) & $65.3 \pm 5.7$ & $62.4 \pm 6.1$ & $59.3 \pm 5.8$ \\
\hline ALT (U/ml) & $18.4 \pm 1.4$ & $16.2 \pm 1.4$ & $18.1 \pm 1.0$ \\
\hline AST (U/ml) & $22.0 \pm 1.7$ & $21.3 \pm 2.1$ & $23.5 \pm 1.6$ \\
\hline
\end{tabular}


Table 3. Effect of hyperhomocysteinemia on markers of carbohydrate metabolism and lipolysis

\begin{tabular}{|l|l|l|l|}
\hline Group & Control & High methionine & $\begin{array}{l}\text { High methionine } \\
\text { vitamin B deficient }\end{array}$ \\
\hline Glucose $(\mathrm{mM})$ & $5.1 \pm 0.2$ & $5.3 \pm 0.3$ & $5.4 \pm 0.2$ \\
\hline Insulin $(\mu \mathrm{U} / \mathrm{ml})$ & $21.4 \pm 2.5$ & $35.6 \pm 3.6^{* *}$ & $38.8 \pm 3.2^{* * *}$ \\
\hline HOMA-IR & $4.85 \pm 0.41$ & $8.42 \pm 0.91^{* * *}$ & $9.31 \pm 1.11^{* * *}$ \\
\hline QUICKI & $0.303 \pm 0.003$ & $0.283 \pm 0.003^{* *}$ & $0.279 \pm 0.002^{* * *}$ \\
\hline NEFA $(\mu \mathrm{M})$ & $372 \pm 21$ & $369 \pm 28$ & $383 \pm 31$ \\
\hline Glycerol $(\mu \mathrm{M})$ & $198 \pm 13$ & $191 \pm 17$ & $195 \pm 16$ \\
\hline
\end{tabular}

HOMA-IR, homeostatic model assessment-insulin resistance index, QUICKI, quantitative insulin sensitivity check index, NEFA, non-esterified fatty acids. ${ }^{* *} p<0.001,{ }^{* * *} p<0.001$ vs. control group.

Table 4. Effect of hyperhomocysteinemia on PON1 activity toward different substrates

\begin{tabular}{|l|l|l|l|}
\hline Group & Control & High methionine & $\begin{array}{l}\text { High methionine } \\
\text { vitamin B deficient }\end{array}$ \\
\hline Paraoxon $(\mathrm{U} / \mathrm{ml})$ & $157 \pm 8$ & $149 \pm 7$ & $109,4 \pm 8^{* * *}$ \\
\hline Phenyl acetate $(\mathrm{U} / \mathrm{ml})$ & $124 \pm 9$ & $119 \pm 6$ & $84,2 \pm 7^{* * *}$ \\
\hline Homocysteine thiolactone $(\mathrm{nmol} / \mathrm{min} / \mathrm{ml})$ & $220 \pm 18$ & $207 \pm 17$ & $127 \pm 11^{* * *}$ \\
\hline
\end{tabular}

${ }^{* *} p<0.001$ versus control group.

did not differ from control. In contrast, PON1 activity toward paraoxon, phenyl acetate and Hcy thiolactone was lower in animals receiving methionine-enriched vitamin B-deficient diet than in control group by $30.3 \%, 32.1 \%$ and $42.5 \%$, respectively (Table 4).

\section{Discussion}

That hHcy may have a detrimental effect on lipid metabolism was first proposed by Werstuck et al (9) who have demonstrated that high Hcy concentrations (1-5 $\mathrm{mM})$ activate sterol regulatory element-binding proteins- 1 and -2 (SREBP- 1 and -2) in cultured hepatoma cells. SREBP-1 and SREBP-2 are transcription factors which stimulate the expression of enzymes involved in fatty acid and cholesterol synthesis, respectively. Consequently, Hcy increased the expression of a rate-limiting enzyme in cholesterol synthesis, 3-hydroxy-3-methylglutarylcoenzyme A (HMG-CoA) reductase, as well as of several more distal enzymes in this pathway such as isopentelypyrophosphate isomerase and farnesylpyrophosphate synthetase. Moreover, Hcy increased the expression of lipogenic enzymes, acetyl-CoA carboxylase and fatty acid synthase. In vivo, plasma cholesterol concentration and hepatic VLDL secretion were higher in hHcy CBS knockout mice (9). In subsequent study (10) hypertriglyceridemia, increased plasma VLDL and apolipoprotein-B100 were also observed in CBS knockout mice. In the rat, administration of methionine in the drinking water for 4 weeks increased hepatic SREBP-2 and HMG-CoA reductase as well as plasma and liver cholesterol concentrations by about $20 \%$ (11).

In the present study plasma cholesterol and triglycerides did not change in hHcy rats which most likely results from different experimental model. In contrast to CBS knockout mice, plasma Hcy is less markedly elevated in our methionine-fed rats; the model which better reflects mild-to-moderate hHcy in humans. In addition, CBS knockout mice suffer from liver steatosis and 
dysfunction which was not observed in methionine-fed rats as evidenced by normal activity of liver enzymes. In many studies in which hHcy was induced by methionine-enriched diets, high methionine doses (up to 1.5-2\% in chow or drinking water) were applied; such doses may also lead to liver damage (12-14). In addition, methionine feeding may affect lipid profile independently of Hcy. Indeed, methionine provides methyl groups for phosphatidylethanolamine N-methyltransferase which converts phosphatidylethanolamine to phosphatidylcholine resulting in the increase in VLDL secretion from the liver (15). In accordance with our results, lower methionine doses $(<1 \%)$ had no effect on plasma total cholesterol, LDL-cholesterol or triglycerides in Sprague-Dawley rats $(16,17)$. Similarly, addition of $0.5 \%$ methionine in the drinking water did not change plasma lipid profile in mice fed normal, "western-type" or atherogenic diet (18). Moreover, hHcy induced by administration of Hcy thiolactone, i.e. not associated with methionine excess, had no effect on plasma lipid profile, cholesterol concentration in the liver, SREBP-2 activity, HMG-CoA reductase or LDL receptor in the rat (19). Taken together, these data suggest that although specific forms of hHcy (genetically determined severe hHcy, very high methionine intake) may affect plasma lipid metabolism, mildto-moderate hHcy has no effect.

Another concern is the link between Hcy and HDL and its integral apolipoprotein, apo A-I. Hcy ( $5 \mathrm{mM})$ inhibited apo A-I synthesis in human hepatoma HepG2 cells (20) and in mouse primary hepatocytes (21). Plasma apo A-I and HDL cholesterol are reduced in heterozygous MTHFR deficient mice $(20,22)$, in CBS knockout mice (23), in apo-E/CBS double knockout mice in comparison to $\mathrm{CBS}^{+/+}$apo- $\mathrm{E}^{-/-}$mice (21) and in homozygous CBS-deficient humans (23). In addition, negative correlation between HDL cholesterol/apo A-I and serum Hcy was observed in patients with ischemic heart disease $(20,24)$. However, in the present study HDL cholesterol and apo A-I were normal in both hyperhomocysteinemic groups. This may be due to (i) lower Hcy concentration than in CBS deficiency, and (ii) species differences - Hcy decreases apo A-I synthesis in human hepatocytes by suppressing the activity of peroxisome proliferator-activated receptor- $\alpha$ (PPAR- $\alpha$ ) which stimulates apo A-I synthesis in humans but not in rodents (25).

We demonstrated that hHcy had no effect on fasting plasma glucose but significantly increased plasma insulin and HOMA index to the similar extent in both groups. These data indicate that $(i)$ even modest Hcy elevation has a detrimental effect on insulin sensitivity and this effect is not dependent on Hcy concentration, and (ii) impaired insulin sensitivity results from high methionine intake/Hcy elevation rather than from vitamin B deficiency. During fasting state, the main metabolic effect of insulin is suppression of hepatic gluconeogenesis and glucose output. When the liver becomes insulin-resistant, pancreatic $\beta$ cells produce more insulin to sustain normoglycemia. Thus, increase in fasting insulin level is the evidence of hepatic insulin resistance (8). In addition, during fasting state insulin suppresses lipolysis in white adipose tissue. However, markers of lipolysis, NEFA and glycerol concentrations, were unchanged in this study, indicating that insulin sensitivity of the liver but not of adipose tissue was reduced. Previously, hHcy induced by Hcy administration has been demonstrated to increase fasting insulin in rats (26) and mice (27). In contrast, feeding mice with high-methionine diet for 3 months has no effect on fasting insulin and glucose as well as on glucose tolerance, but insulin secretion during oral glucose tolerance test was elevated, suggesting post-prandial (decrease in insulin-induced glucose disposal) rather than fasting insulin resistance (28). However, in the same study, pretreatment of primary mouse hepatocytes with $0.1 \mathrm{mM} \mathrm{Hcy}$ for 6 hours abolished suppressing effect of insulin on glucose output, which supports our results. Moreover, the expression of a rate-limiting enzyme of gluconeogenesis, phosphoenolpyruvate carboxykinase (PEPCK), was higher in hyperhomocysteinemic mice at both mRNA and protein level (28). In vitro, Hcy increased PEPCK expression in mouse hepatocytes, and inhibitor of protein kinase A abolished the effect of Hcy on PEPCK expression and insulin-induced inhibition of glucose output. These data indicate that hHcy impairs the effect of insulin on hepatic gluconeogenesis. Moreover, Hcy thiolactone inhibits the effect of insulin on tyrosine phosphorylation of insulin receptor substrate-1 in cultured human hepatocytes (29). Apart from direct effect on hepatocytes, hHcy may impair insulin sensitivity by modifying adipokines production. For example, hHcy has been demonstrated to stimulate resistin (27) and inhibit adiponectin (30) secretion. The mechanism of insulin resistance induced by hHcy in the present study remains to be established.

PON1 is synthesized in the liver and circulates attached to plasma HDL. Lipid composition of HDL as well as its integral apolipoprotein, apo A-I, are essential for PON1 transfer from hepatocytes to lipoproteins and its stability and activity. The inverse correlation between Hcy concentration and PON1 activity was reported in clinical studies (31-34). In addition, PON1 expression in the liver is increased in patients with Down syndrome who have high CBS level (the $c b$ s gene is located on chromosome 21) and low plasma Hcy (35). Hepatic PON1 expression and activity are dramatically reduced in homozygous CBS knockout mice $(36,37)$, however, this effect most likely results from liver dysfunction and/or HDL and apo A-I deficiency. CBS knockout mice with transient transgenic expression of the human enzyme, 
in which Hcy concentration is comparable to CBS knockout animals but liver function is intact, exhibit normal PON1 activity toward paraoxon and only slightly reduced (-17\%) activity toward phenyl acetate (23). In untreated or poorly compliant CBS deficient patients (plasma Hcy 160-310 $\mu \mathrm{M}$ ) PON1 activity toward paraoxon did not differ from normohomocysteinemic healthy subjects, but its activity toward phenyl acetate was by $30 \%$ lower (23). These data suggested that hHcy has little effect on PON1 in the absence of liver dysfunction.

However, PON1 expression in the liver is also reduced in heterozygous $\mathrm{CBS}^{+/-}$mice fed a high-methionine diet (but not in $\mathrm{CBS}^{+/-}$mice fed a regular diet), in which liver function is not impaired (37). The polyphenolic compound, catechin (38), as well as red wine polyphenols (39) reduced plasma Hcy concentration and increased PON1 expression in the liver of heterozygous $\mathrm{CBS}^{+/-}$mice fed a high-methionine diet, whereas another polyphenol, quercetin, had no effect on either Hcy or PON1 (38). Surprisingly, one of the most prevalent polyphenol of the red wine, resveratrol, administered alone, increased plasma Hcy and further reduced PON1 expression and activity in methioninefed $\mathrm{CBS}^{+/-}$mice $(40)$. Ferretti et al $(41,42)$ have demonstrated that incubation of HDL isolated from healthy normolipidemic subjects with Hcy thiolactone decreases PON1 activity. Clinical studies have indicated that mild hHcy $(\sim 20 \mu \mathrm{M})$ has no effect on PON1 status but more sever hHcy $(\sim 70 \mu \mathrm{M})$ is associated with PON1 deficiency (43). We demonstrated that high-methionine vitamin B-deficient but not high-methionine vitamin B-sufficient diet results in the deficiency of plasma PON1 despite normal HDL and apo A-I levels. Together with clinical observations (43) and studies in mice with different Hcy concentrations (44), these results indicate that PON1 activity decreases above certain Hcy level even if liver function, HDL concentration and apo A-I are normal. If this effect results from direct inhibition of plasma PON1 by Hcy (42) or other mechanisms such as specific suppression of enzyme synthesis in the liver, remains to be established.

In conclusion, we have demonstrated that hHcy induced in the rat by high-methionie or high-methionine vitamin B-deficient diets has no effect on plasma lipid concentration as well as on apolipoprotein A-I. Both high-methionine diets increased fasting plasma insulin suggesting impaired effect of this hormone on hepatic glucose output. Hyperhomocysteinemia had no effect on non-esterified fatty acids or glycerol indicating that antilipolytic effect of insulin was intact. Plasma PON1 activity was normal in high-methionine fed group but was reduced in high-methionine vitamin B-deficient group. Decrease in insulin sensitivity and PON1 deficiency may contribute to detrimental effects of hHcy on cardiovascular system.

\section{Acknowledgements}

This study was supported by EU Project "The equipment of innovative laboratories doing research on new medicines used in the therapy of civilization and neoplastic diseases" within the Operational Program Development of Eastern Poland 2007 - 2013, Priority Axis I Modern Economy, Operations I.3 Innovation Promotion.

\section{References}

1. Castro R, Rivera I, Blom HJ, Jakobs C, Tavares de Almeida I. Homocysteine metabolism, hyperhomocysteinaemia and vascular disease: an overview. J Inherit Metab Dis 2006; 29: 3-20.

2. Bełtowski J, Tokarzewska D. Adipose tissue and homocysteine metabolism. Biomed Rev 2010; 20: 7-15.

3. Steed MM, Tyagi SC. Mechanisms of cardiovascular remodeling in hyperhomocysteinemia. Antioxid Redox Signal 2011; 15: 1927-1943.

4. Perła-Kaján J, Jakubowski H. Paraoxonase 1 and homocysteine metabolism. Amino Acids 2012 (in press).

5. De Vriese AS, Blom HJ, Heil SG, Mortier S, Kluijtmans LA, Van de Voorde J, Lameire NH. Endothelium-derived hyperpolarizing factor-mediated renal vasodilatory response is impaired during acute and chronic hyperhomocysteinemia. Circulation 2004; 109: 2331-2336.

6. Beltowski J, Wójcicka G, Jakubowski H. Modulation of paraoxonase 1 and protein $\mathrm{N}$-homocysteinylation by leptin and the synthetic liver X receptor agonist T0901317 in the rat. $J$ Endocrinol 2010; 204: 191-198.

7. Bełtowski J, Atanassova P, Chaldakov GN, JamrozWiśniewska A, Kula W, Rusek M. Opposite effects of pravastatin and atorvastatin on insulin sensitivity in the rat: role of vitamin D metabolites. Atherosclerosis 2011; 219: 526-531.

8. Muniyappa R, Chen H, Muzumdar R, Einstein FH, Yan X, Yue $\mathrm{LQ}$, et al. Comparison between surrogate indexes of insulin sensitivity/resistance and hyperinsulinemic euglycemic clamp estimates in rats. Am J Physiol Endocrinol Metab 2009; 297: E1023-1029.

9. Werstuck GH, Lentz SR, Dayal S, Hossain GS, Sood SK, Shi YY, et al. Homocysteine-induced endoplasmic reticulum stress causes dysregulation of the cholesterol and triglyceride biosynthetic pathways. J Clin Invest 2001; 107: 1263-1273.

10. Namekata K, Enokido Y, Ishii I, Nagai Y, Harada T, Kimura H. Abnormal lipid metabolism in cystathionine beta-synthase-deficient mice, an animal model for hyperhomocysteinemia. J Biol Chem 2004; 279: 52961-52969.

11. Woo CW, Siow YL, Pierce GN, Choy PC, Minuk GY, Mymin D. Hyperhomocysteinemia induces hepatic cholesterol 
biosynthesis and lipid accumulation via activation of transcription factors. Am J Physiol Endocrinol Metab 2005; 288 : E1002-1010.

12. Woo CW, Prathapasinghe GA, Siow YL. Hyperhomocysteinemia induces liver injury in rat: Protective effect of folic acid supplementation. Biochim Biophys Acta 2006; 1762: 656-665.

13. Wu N, Siow YL, OK. Induction of hepatic cyclooxygenase-2 by hyperhomocysteinemia via nuclear factor-kappaB activation. Am J Physiol Regul Integr Comp Physiol 2009; 297: R1086-1094.

14. Yalçinkaya S, Unlüçerçi Y, Giriş M, Olgaç V, DoğruAbbasoğlu S, Uysal M. Oxidative and nitrosative stress and apoptosis in the liver of rats fed on high methionine diet: protective effect of taurine. Nutrition 2009; 25: 436-444.

15. Obeid R, Herrmann W. Homocysteine and lipids: S-adenosyl methionine as a key intermediate. FEBS Lett 2009; 583: 12151225.

16. Morita H, Saito Y, Kurabayashi M, Nagai R. Diet-induced mild hyperhomocysteinemia and increased salt intake diminish vascular endothelial function in a synergistic manner. J Hypertens 2002; 20: 55-62.

17. Hirche F, Schroder A, Knoth B, Stangl GI, Eder K. Methionine-induced elevation of plasma homocysteine concentration is associated with an increase of plasma cholesterol in adult rats. Ann Nutr Metab 2006; 50: 139-146.

18. Zhou J, Werstuck GH, Lhoták S, Shi YY, Tedesco V, Trigatti $\mathrm{B}$, et al. Hyperhomocysteinemia induced by methionine supplementation does not independently cause atherosclerosis in C57BL/6J mice. FASEB J 2008; 22: 2569-2578.

19. Stangl GI, Weisse K, Dinger C, Hirche F, Brandsch C, Eder K. Homocysteine thiolactone-induced hyperhomocysteinemia does not alter concentrations of cholesterol and SREBP-2 target gene mRNAs in rats. Exp Biol Med (Maywood) 2007; 232: 81-87.

20. Mikael LG, Genest J Jr, Rozen R. Elevated homocysteine reduces apolipoprotein A-I expression in hyperhomocysteinemic mice and in males with coronary artery disease. Circ Res 2006; 98: 564-571.

21. Liao D, Tan H, Hui R, Li Z, Jiang X, Gaubatz J, et al. Hyperhomocysteinemia decreases circulating high-density lipoprotein by inhibiting apolipoprotein A-I protein synthesis and enhancing HDL cholesterol clearance. Circ Res 2006; 99: 598-606.

22. Schwahn BC, Wang XL, Mikael LG, Wu Q, Cohn J, Jiang H, et al. Betaine supplementation improves the atherogenic risk factor profile in a transgenic mouse model of hyperhomocysteinemia. Atherosclerosis 2007; 195: e100-107.
23. Jiang H, Stabler SP, Allen RH, Maclean KN. Altered expression of apoA-I, apoA-IV and PON-1 activity in CBS deficient homocystinuria in the presence and absence of treatment: Possible implications for cardiovascular outcomes. Mol Genet Metab 2012 (in press).

24. Xiao Y, Zhang Y, Lv X, Su D, Li D, Xia M, et al. Relationship between lipid profiles and plasma total homocysteine, cysteine and the risk of coronary artery disease in coronary angiographic subjects. Lipids Health Dis 2011; 10: 137.

25. Vu-Dac N, Chopin-Delannoy S, Gervois P, Bonnelye E, Martin G, Fruchart JC, et al. The nuclear receptors peroxisome proliferator-activated receptor alpha and Rev-erb alpha mediate the species-specific regulation of apolipoprotein A-I expression by fibrates. J Biol Chem 1998; 273: 25713-25720.

26. Golbahar J, Aminzadeh MA, Kassab SE, Omrani GR: Hyperhomocysteinemia induces insulin resistance in male Sprague-Dawley rats. Diabetes Res Clin Pract 2007, 76:1-5.

27. Li Y, Jiang C, Xu G, Wang N, Zhu Y, Tang C, Wang X. Homocysteine upregulates resistin production from adipocytes in vivo and in vitro. Diabetes 2008; 57: 817-27.

28. Yu X, Huang Y, Hu Q, Ma L. Hyperhomocysteinemia stimulates hepatic glucose output and PEPCK expression. Acta Biochim Biophys Sin 2009; 41: 1027-1032.

29. Najib S, Sánchez-Margalet V. Homocysteine thiolactone inhibits insulin-stimulated DNA and protein synthesis: possible role of mitogen-activated protein kinase (MAPK), glycogen synthase kinase-3 (GSK-3) and p70 S6K phosphorylation. J Mol Endocrinol 2005; 34: 119-126.

30. Song Z, Zhou Z, Deaciuc I, Chen T, McClain CJ. Inhibition of adiponectin production by homocysteine: a potential mechanism for alcoholic liver disease. Hepatology 2008; 47: 867-879.

31. Chassé JF. Inverse correlation between phenylacetate hydrolase activity of the serum PON1 protein and homocysteinemia in humans. Thromb Haemost 2005; 93: 182-183.

32. Murphy MM, Marsillach J, Camps J, Fernández-Ballart J, Mackness B, Mackness M, et al. Influence of PON1 polymorphisms on the association between serum paraoxonase 1 and homocysteinemia in a general population. Clin Chem 2006; 52: 781-782.

33. Kerkeni M, Addad F, Chauffert M, Chuniaud L, Miled A, Trivin F, et al. Hyperhomocysteinemia, paraoxonase activity and risk of coronary artery disease. Clin Biochem 2006; 39: 821-825.

34. Karikas GA, Kriebardis A, Samara I, Schulpis K, Papachristodoulou M, Fytou-Pallikari A. Serum homocysteine levels and paraoxonase 1 activity in preschool aged children in Greece. Clin Chem Lab Med 2006; 44: 623-627. 
35. Janel N, Christophe O, Ait Yahya-Graison E, Hamelet J, Paly E, Prieur M, et al. Paraoxonase-1 expression is up-regulated in Down syndrome fetal liver. Biochem Biophys Res Commun 2006; 346: 1303-1306.

36. Robert K, Chassé JF, Santiard-Baron D, Vayssettes C, Chabli A, Aupetit J, et al. Altered gene expression in liver from a murine model of hyperhomocysteinemia. J Biol Chem 2003; 278: 31504-31511.

37. Janel N, Robert K, Chabert C, Ledru A, Gouédard C, Barouki $\mathrm{R}$, et al. Mouse liver paraoxonase-1 gene expression is downregulated in hyperhomocysteinemia. Thromb Haemost 2004; 92: 221-222.

38. Hamelet J, Demuth K, Dairou J, Ledru A, Paul JL, Dupret $\mathrm{JM}$, et al. Effects of catechin on homocysteine metabolism in hyperhomocysteinemic mice. Biochem Biophys Res Commun 2007; 355: 221-227.

39. Noll C, Hamelet J, Matulewicz E, Paul JL, Delabar JM, Janel N. Effects of red wine polyphenolic compounds on paraoxonase-1 and lectin-like oxidized low-density lipoprotein receptor-1 in hyperhomocysteinemic mice. J Nutr Biochem 2009; 20: 586-596.
40. Noll C, Hamelet J, Ducros V, Belin N, Paul JL, Delabar JM, et al. Resveratrol supplementation worsen the dysregulation of genes involved in hepatic lipid homeostasis observed in hyperhomocysteinemic mice. Food Chem Toxicol 2009; 47: 230-236.

41. Ferretti G, Bacchetti T, Marotti E, Curatola G. Effect of homocysteinylation on human high-density lipoproteins: a correlation with paraoxonase activity. Metabolism 2003; 52: 146-151.

42. Ferretti G, Bacchetti T, Masciangelo S, Bicchiega V. Effect of homocysteinylation on high density lipoprotein physicochemical properties. Chem Phys Lipids 2010; 163: 228-235.

43. Holven KB, Aukrust P, Retterstøl K, Otterdal K, Bjerkeli V, Ose L, et al. The antiatherogenic function of HDL is impaired in hyperhomocysteinemic subjects. J Nutr 2008; 138: 2070-2075.

44. Hamelet J, Aït-Yahya-Graison E, Matulewicz E, Noll C, Badel-Chagnon A, Camproux AC, et al. Homocysteine threshold value based on cystathionine beta synthase and paraoxonase 1 activities in mice. Eur J Clin Invest 2007; 37: 933-938. 